\title{
Evaluation of mixture-fraction-based turbulent-reaction-rate model assumptions for high-pressure reactive flows
}

\author{
Josette Bellan $^{\mathrm{a}, \mathrm{b}, *}$ \\ ${ }^{a}$ California Institute of Technology, Pasadena, CA 91125, USA \\ ${ }^{\mathrm{b}}$ Jet Propulsion Laboratory, California Institute of Technology, Pasadena, CA \\ 91109, USA
}

\begin{abstract}
Several assumptions of atmospheric-pressure (atmospheric- $p$ ) single-phase turbulent reaction rate models are examined for high- $p$ reactive flows having turbulent characteristics. The study uses a Direct Numerical Simulation (DNS) database described elsewhere [1]. This database was obtained with a model combining multi-species mixing under high- $p$ conditions, a real-gas equation of state (EOS) and a single-step chemical reaction. The database, created in the configuration of a temporal mixing layer, probes the effect of the initial Reynolds number, $\mathrm{Re}_{0}$, of the initial pressure, $p_{0}$, and of the initial composition of the two mixing-layer streams. The reaction is initiated in a turbulent flow and in each simulation the computations are pursued past a time when a maximum average-volumetric $p$ is attained, $t_{p p}^{*}$. The examination of the vorticity and enstrophy equations at a time before reaction initiation and also at $t_{p p}^{*}$ highlights the pivotal role of the high density-gradient magnitude regions [1] in producing turbulence and the crucial role at $t_{p p}^{*}$ of the baroclinic effect representing the misalignments of gradients of thermodynamic variables. Compared to the classical mixture fraction equation, the results show that the mixture fraction obeys an equation in which there is an additional diffusion term having a larger r.m.s. magnitude than that of the mixture-fraction typical diffusion term. An assessment of the Conditional Source-term Estimation (CSE) model assumptions found that, using an accurate mixture fraction probability density function (PDF), one can obtain a very good representation of the turbulent reaction rate in the most intense reaction regions, however the quality of the predictions of CSE deteriorated significantly when a common model for the PDF, the $\beta$-PDF, was used despite the $\beta$-PDF being constructed with the accurate moments extracted from the DNS. In the regions of minimal reaction, the CSE model using the exact PDF extracted from the DNS does not provide an accurate representation of the turbulent reaction rate, a fact which is attributed to the combined effect of lack of correlation between fluctuations of the reaction rate and of the mixture fraction, and to the strong correlation of the thermodynamic variables through the real-gas EOS in regions of colder and denser fluid.
\end{abstract}

Preprint submitted to Combustion and Flame

7 February 2017

(C) 2017. This manuscript version is made available under the Elsevier user license

http://www.elsevier.com/open-access/userlicense/1.0/ 
Key words: high-pressure turbulent reaction rate model; turbulence production in high-pressure turbulent flows; Direct Numerical Simulation

\section{Introduction}

The modeling of the turbulent reaction rate under high-pressure (high- $p$ ) conditions has so far utilized the formulations developed for single-phase atmospheric$p$ reactive flows. A recent review of most utilized turbulent reaction models [2] classified these models into three categories: probability density function (PDF) models [3], Linear Eddy Model models (e.g. [4]) and mixture-fraction type models (e.g. $[5,6,7,8]$ ). To date, PDF models have not been used to simulate high- $p$ reactive flow presumably because they do not yet embed a model portraying the crucial complexities of high- $p$ multi-species diffusion [1] such as uphill diffusion [9] which may affect phase change. The Linear Eddy Model has only been considered for situations where the mass diffusion is assumed to be of Fickian type (e.g. [4]) and the Kolmogorov theory is valid in predicting the needed information of the stochastic rearrangements at the smallest scale; the more recent One-Dimensional Turbulence model [10] is a variation of the LEM with the difference that a momentum equation is used to provide the frequency of the rearrangements. Preponderantly, turbulent reaction rate models used in Large Eddy Simulation (LES) rely on a definition of a variable denoted as the mixture fraction, $\varsigma$, and employ various assumptions to arrive at an expression for the reaction rate; if such models should be considered for LES of high- $p$ turbulent combustion, the validity of the assumptions embedded in them must be evaluated. There is a variety of definitions of $\varsigma$ but a simple classification of these definitions shows two categories: (1) definitions relying on the use of the fuel and oxidizer mass fractions (e.g. [5,7]) and (2) definitions relying on the utilization of the elements rather than the species (e.g. $[6,11])$. Mathematically, the $\varsigma$ definitions are equivalent. Flamelet models [8], the Conditional Moment Closure (CMC) method (e.g. [12,13,14]) or the Conditional Source-term Estimation (CSE) method $[15,16]$ are all inter-related and using one of these definitions or the mixture fraction (e.g. [7]). Because the traditional combustion models adopt Fickian diffusion or an approximation of the mass-diffusion matrix that makes it diagonal $[11,17]$, any deviation from $\varsigma$ obeying an equation of the form

$$
\rho \frac{D \varsigma}{d t}=\nabla \cdot\left(\rho D_{\varsigma} \nabla \varsigma\right)
$$

\footnotetext{
* Corresponding author. Tel: (818) 354-6959, Fax: (818) 393-6682.

Email address: Josette.Bellan@jpl.nasa.gov (Josette Bellan).
} 
where $\rho$ is the density, $D / d t$ is the material derivative operator with $t$ being the physical time, and where $D_{\varsigma}$ is a diffusion coefficient, has been erroneously considered to be in a non-conservative form [11]; from the mathematical viewpoint there could be in Eq. (1) additional diffusion-type terms depending on variables other than $\varsigma$ that would mathematically still make the equation conservative, but some adopted terminology in the literature (e.g. [11]) considers that these are source terms as far as the conservation of $\varsigma$ is concerned, although a better terminology would be to call the latter type of equation "conservative but not self-contained". Experimental observations of atmospheric- $p$ flames have been reported [18] showing that according to the definition of a conserved scalar of Ref. [11] using Eq. (1), the atomic mass is not a conserved quantity even for some premixed flames, a fact which is shown through simulations to be due to differential diffusion. More than three decades ago, the importance of differential diffusion in atmospheric- $p$ flows has been raised for the mixture fraction defined based on elements [19] and it has numerically been shown to be non-negligible. When differential diffusion is considerable, as it could be in high- $p$ combustion, the $\varsigma$ equation cannot be solved independently of the equations for the other dependent variables so that the benefit of this element-based $\varsigma$ definition is not fulfilled for the goal of ultimately recovering the mass fractions from the solution of the equations. Thus, adopting turbulent reaction rate models already shown to be based on arguable formulations at atmospheric- $p$ conditions becomes risky for high- $p$ situations.

There is recent evidence [20] that there are difficulties with the typical flamelet model, a fact which prompted its adaptation to unsteady flames and its utilization in a higher-order form wherein departures from the classical assumption (that the reaction zone thickness is much smaller than a distance based on the flame curvature) are taken into account. The interaction of flamelets implied in the higher-order flamelet model has been interpreted [21] as scalar diffusion occurring orthogonally to the mixture-fraction gradient. In the present study we show that for high- $p$ flows with turbulent characteristics, the mixture fraction, while still obeying a conservation equation, no longer satisfies the more restrictive self-contained conservation equation prevailing in the combustion literature (e.g. [11]), i.e. Eq. (1), and that this fact can also be traced to diffusion aspects - here differential diffusion. The CMC and CSE models have in common with the flamelet model the necessity of knowing the mixture fraction. Moreover, in the CMC model it is assumed that the fluctuations of the reactive scalars at a given mixture fraction value must be small relative to mean concentrations conditioned on the mixture fraction [22], but it is conceivable that the presence of differential diffusion may lead to larger conditional fluctuations, an aspect which has not yet been investigated.

This investigation is devoted to the evaluation, in the realm of high- $p$ reactive flows, of several assumptions used in $\varsigma$-based models; the interest is to understand the coupling among a generic reaction, high- $p$ transport and high- $p$ 
thermodynamics. To conduct this evaluation, a Direct Numerical Simulation (DNS) database is utilized to provide the necessary information for the study. Since the full formulation used to create the database has been described elsewhere [1], only its crucial features are succinctly provided in Section 2. The database is described in Section 3. Then, in Section 4, results from a previous analysis of this database [1] are recalled. The analysis presented in Section 5 first addresses turbulence development in the flow, then the focus is on the mixture fraction and finally on the examination of the primary assumption of the CSE model as a typical example of an advanced turbulent reaction rate model developed for atmospheric- $p$ combustion. A summary and conclusions are offered in Section 6.

\section{Description of the governing equations}

The governing equations are the conservation equations - continuity, momentum, species and total energy - complemented by the Peng-Robinson (PR) real-gas equation of state (EOS). In the conservation equations, the fluxes of species and heat are written according to fluctuation-dissipation theory [23]. The species fluxes consist of the multicomponent species mass diffusion with a fully populated diffusion matrix and the Soret effect; the heat flux contains the Fourier term, Dufour effects and the enthalpy transported by the species fluxes. All transport properties - viscosity, mass diffusion coefficients, thermal diffusions coefficients and thermal conductivity - are computed using highpressure-valid mixing rules [24], and thus transport properties are functions of all thermodynamic variables, $\left(p, T, X_{\alpha}\right)$ where $T$ is the temperature and $X_{\alpha}$ is the mole fraction of species $\alpha$. The PR EOS is well known to be inaccurate for species other than hydrocarbons and therefore the volume correction of [25] computed from a reference Gibbs free energy is used to enhance its accuracy to the same level as the Lee-Kesler EOS. Details on the entire formulation can be found in [1].

The reaction is initiated in the cold-ignition regime, and the chemistry is represented by a generic single-step reaction

$$
\mathrm{C}_{7} \mathrm{H}_{16}+11 \mathrm{O}_{2}+\mathrm{N}_{2} \rightarrow 7 \mathrm{CO}_{2}+8 \mathrm{H}_{2} \mathrm{O}+\mathrm{N}_{2}
$$

of rate

$$
\dot{\omega}=A T^{n} \frac{\rho^{a+b}}{m_{\mathrm{C}_{7} \mathrm{H}_{16}} m_{\mathrm{O}_{2}}} Y_{\mathrm{C}_{7} \mathrm{H}_{16}}^{a} Y_{\mathrm{O}_{2}}^{b} \exp \left(-E_{a} / R_{u} T\right)
$$

where $\rho$ is the density, $Y_{\alpha}=X_{\alpha} m_{\alpha} / m, m_{\alpha}$ is the molar mass of species $\alpha$, $m$ is the mixture molar mass, $m=\sum_{\gamma=1}^{N} m_{\gamma} X_{\gamma}, E_{a}$ is the activation energy, $R_{u}$ is the universal gas constant and $A$ is the pre-exponential constant. To ensure that the minimum ignition delay time occurred in rich mixtures when 
the oxidizer $T$ is larger than the fuel $T$, in concert with the finding of other investigators $[26,27,28]$, the values $a=b=1$ were chosen. As in [29], $E_{a}=$ $30 \mathrm{kcal} / \mathrm{mol}$ and $n=0$, whereas $A=9.6 \times 10^{6} \mathrm{~cm}^{3}(\mathrm{~mol} \times \mathrm{s})^{-1}$ here to avoid abrupt ignition. Of note, with $a=b=1, \dot{\omega} \propto \rho^{2}$ and thus $\dot{\omega}$ strongly depends on $p$. The utilization of a single-step reaction is well-established for DNS of reactive flow fundamental studies, e.g. [30,31].

\section{The database}

The governing equations were solved in a temporal mixing layer configuration using Direct Numerical Simulation (DNS) meaning that all scales overwhelmingly contributing to the dissipation were resolved. Periodic boundary conditions were used in the homogeneous directions - streamwise direction, $x_{1}$, and the spanwise direction, $x_{3}$ - and non-reflecting boundary conditions were utilized in the cross-stream direction, $x_{2}$. The mixing layer was composed of a relatively hotter upper stream and a lower stream moving counter current with respect to the upper stream. The lower stream initially contained $\mathrm{C}_{7} \mathrm{H}_{16}$ and may also have contained $\mathrm{CO}_{2}$ and $\mathrm{H}_{2} \mathrm{O}$. The upper stream initially contained air which was sometime vitiated with $\mathrm{CO}_{2}$ and $\mathrm{H}_{2} \mathrm{O}$ The initial $\mathrm{CO}_{2}$ and $\mathrm{H}_{2} \mathrm{O}$ in the composition simulate Exhaust Gas Recirculation (EGR). As typical of DNS initial conditions, the value of the initial Reynolds number was based on a reference molecular viscosity rather than the physical molecular viscosity; to ensure that the Prandtl number, Pr, and the species Schmidt number, $S c_{\alpha}$, retain their realistic physical values, the physical values of the molecular conductivity and molecular mass diffusivities were scaled by the ratio between the reference viscosity and physical viscosity (see [1], section 4.1). Initially, the layer hosted four spanwise vortices which, through a double merging, formed an ultimate vortex in which small scales proliferated. Eventually a transitional (non-dimensional) time $t_{t r}^{*}$ was reached at which spectra based on velocity fluctuations and on fluctuations of thermodynamic variables show a smooth behavior. The spectra also show no accumulation of energy at the smallest scales, indicating the DNS good resolution. The spectra of the thermodynamic variables are of interest because it has been shown that $\operatorname{Pr}>1$ in supercritical $[32,33]$ and in high- $p$ flows [34], and that the effective speciesspecific Schmidt number, $S c_{e f f, \alpha}>1$ in substantial regions of the flow [34], all of which indicates that the thermodynamic scales are smaller than the Kolmogorov scale.

The reaction is initiated at $t_{t r}^{*}$, and therefore ignition occurs in a flow with turbulent characteristics. Several DNS realizations were obtained to study the influence of the initial Reynolds number, $\mathrm{Re}_{0}$, of the initial pressure, $p_{0}$, and of EGR. Tables 1 and 2 show the initial conditions in detail. In Table $1 \rho_{U}$ and $\rho_{L}$ are the mixture initial densities, where subscripts $U$ and 
$L$ label the upper and lower streams respectively. During mixing and up to $t_{t r}^{*}$, the volumetric-average pressure remained approximately constant [34] but once ignition occurred, due to the heat release the volumetric-average pressure began to increase and eventually a volumetrically-averaged $p$ peak was obtained [1,35]; that (non-dimensional) time was labeled $t_{p p}^{*}$ and the solution at that time is representative of full combustion. Since high- $p$ flows are not ergodic $[36,37]$ and the mixing layers are inherently unsteady, the analysis was primarily performed at $t_{p p}^{*}$ and common aspects of behavior among the simulations were considered representative of the high- $p$ behavior. Table 1 also lists the momentum-thickness based Reynolds numbers at $t_{t r}^{*}$ and $t_{p p}^{*}$ where $\operatorname{Re}_{m} \equiv \operatorname{Re}_{0} \delta_{m} / \delta_{\omega, 0}$ with

$$
\delta_{m}=\frac{\int_{x_{2}, \min }^{x_{2}, \max }\left[\left\langle\rho u_{1}\right\rangle_{x_{2}, \max }-\left\langle\rho u_{1}\right\rangle\right]\left[\left\langle\rho u_{1}\right\rangle-\left\langle\rho u_{1}\right\rangle_{x_{2}, \min }\right] d x_{2}}{\left(\left\langle\rho u_{1}\right\rangle_{x_{2}, \max }-\left\langle\rho u_{1}\right\rangle_{x_{2}, \min }\right)^{2}},
$$

where $x_{2, \min }=-L_{2} / 2.5, x_{2, \max }=x_{2, \min }+L_{2}$, and \langle\rangle symbolizes averages over homogeneous $\left(x_{1}, x_{3}\right)$ planes and $L_{2}$ is the cross-stream domain size listed in Table 1 and $u$ is the velocity. The value of $L_{2}$ is simulation dependent to ensure that the ultimate vortex does not interfere with the domain boundary. By definition, $\delta_{\omega, 0} \equiv \Delta U_{0} /\left(\partial u_{0} / \partial x_{2}\right)_{\max }$ is the initial vorticity thickness computed by using $u_{0}$ which is the $\left(x_{1}, x_{3}\right)$ planar average of the initial velocity in the streamwise direction; $\Delta U_{0}=U_{U}-U_{L}$ is the initial velocity difference across the layer. The non-dimensional time is defined as $t^{*} \equiv t \Delta U_{0} / \delta_{\omega, 0}$.

The properties of the five species in the mixing layer are listed in Table 3.

\section{Brief description of previous results obtained from analyzing the database}

As a precursor to the study reported below, several pertinent results from a previous [1] study are first briefly recalled.

When reaction occurs, the growth of $\delta_{m} / \delta_{\omega, 0}$ accelerates compared to that taking place during mixing; this acceleration is attributed to the partial backscatter of the thermodynamic energy which is released during combustion at the smallest scales. Indeed, this released energy is partially dissipated and partially backscattered; see detailed explanation elsewhere [1]. Examination of the reaction at $t_{p p}^{*}$ shows that the reaction is established in the oxidizer stream and is of a partially-premixed type, with the highest reaction rate occurring in the diffusion flame which is surrounded by patches of premixed burning having modest reaction rates compared to the diffusion flame; some premixed reaction patches occur in the fuel stream [1]. A common feature of all simulations is the formation of high $|\nabla \rho|$ magnitude (HDGM) regions which result 
from the species diffusional mixing. The HDGM observation is entirely consistent with experiments conducted under high- $p$ fully turbulent flow conditions $[38,39,40,41]$ and detected [42] in the Engine Combustion Network Spray A Sandia data [43] that was obtained at an ambient $p$ of $60 \mathrm{~atm}$ and ambient $T$ of $900 \mathrm{~K}$ (compare with the free-stream conditions of Tables 1 and 2). This latter data is described on the Sandia web site [43] as "The Spray A condition is a low-temperature combustion condition relevant to engines that use moderate EGR" which is entirely consistent with the composition listed in Table 2. Another important feature of the flows obtained in the DNS realizations [1] is the occurrence of uphill diffusion which is a molecular-induced effect (unlike the counter-gradient effect in atmospheric- $p$ turbulent flows that is of convective origin) and occurs due to interactions among (rather than between) species. Uphill diffusion is measured by an effective species-specific diffusion coefficient which includes molecular diffusivities (i.e. mass diffusivities, Soret diffusion and barodiffusion) intertwined with the effect of thermodynamicvariable gradients (see [34] for details). Uphill diffusion only occurred for the trace species $\mathrm{CO}_{2}$ and $\mathrm{H}_{2} \mathrm{O}$ and for the EGR cases because it is most operative during mixing under relatively cold conditions, i.e. when $\rho$ is relatively large. Thus, at early times, uphill diffusion dominates for both $\mathrm{H}_{2} \mathrm{O}$ and $\mathrm{CO}_{2}$, independent of the value of $|\nabla \rho|$, this being due to the mixing of these species with the cold and dense fuel. For $\mathrm{H}_{2} \mathrm{O}$, at $t_{p p}^{*}$, in the regions of largest $|\nabla \rho|$ value, the propensity of regular diffusion is sometimes larger than that of uphill diffusion whereas this does not occur for $\mathrm{CO}_{2}$, this difference being attributed to the disparate size of their molecules and molar masses which influence the ability of the molecule to diffuse among other molecules in a dense gas. This phenomenon is thus typically initiated in the relatively low $T$ regime associated with cold ignition, but once initiated, the results showed that it persists during combustion. Uphill diffusion produces density gradients which act as a solid boundary [44] and produce turbulence. The results showed that $|\nabla \rho|$ was larger in regions of higher reaction rate, which expectably was strongly dependent on $p_{0}$. In the $|\nabla \rho|$ regions, the range of reaction rates was independent of the $\operatorname{Re}_{0}$ value, but the $|\nabla \rho|$ magnitude at fixed reaction rate increased at larger $\operatorname{Re}_{0}$. For the present conditions, the range of the reaction rates was approximately similar for the simulation conducted at the higher $p_{0}$ and for the simulation devoid of EGR effects, however at otherwise same initial conditions the $|\nabla \rho|$ magnitude was larger for the higher- $p$ simulation, consistent with the reduced effective diffusivity at larger $p_{0}$ [34].

\section{Results}

The present results are organized as follows: In section 5.1 the vortical characteristics of the layer are addressed since they have a strong impact on the 
reaction. A study assessing assumptions used in atmospheric- $p$ advanced models of the turbulent reaction rate is presented in section 5.2.

Similar to the previous study [1], since ergodicity cannot be assumed, the strategy is here to focus on the common aspects of the results for all DNS listed in Table 1 and consider as being representative of the situation studied. This approach is reinforced by experimental and theoretical information showing that the flows of present interest have distinct inhomogeneities $[34,38,39,45,46]$, meaning that statistical averages, which smear these aspects, may not be representative of the local aspect of the flow since these local inhomogeneities influence turbulence production $[34,44]$ and possible phase separation due to uphill diffusion $[37,47,48]$. Thus, the analysis is focussed on the state obtained at $t_{p p}^{*}$ although earlier states before reaction initiation are also examined to comparatively understand the influence of the reaction.

\subsection{Vortical characteristics}

Turbulence and vorticity are intertwined concepts [49]. The classical view is that turbulence originates from vorticity. It is well-known that vorticity forms at material boundaries and is transported through the flow; much less discussed is the fact that turbulence may form at $\nabla \rho$ boundaries which are not necessarily material interfaces $[34,44]$. To identify the phenomena locally governing vorticity production, the focus is on the vorticity and the enstrophy equations

$$
\begin{gathered}
\frac{D \boldsymbol{\omega}}{D t}=(\boldsymbol{\omega} \cdot \nabla) \mathbf{u}-(\nabla \cdot \mathbf{u}) \boldsymbol{\omega}-\nabla\left(\frac{1}{\rho}\right) \times \nabla p+\nabla \times\left(\frac{1}{\rho} \nabla \cdot \overline{\bar{\sigma}}\right) \\
\frac{D\left(\boldsymbol{\omega}^{2}\right)}{D t}=2 \boldsymbol{\omega} \cdot(\boldsymbol{\omega} \cdot \nabla) \mathbf{u}-\mathbf{2}(\nabla \cdot \mathbf{u}) \boldsymbol{\omega}^{2}-2 \boldsymbol{\omega} \cdot \nabla\left(\frac{1}{\rho}\right) \times \nabla p+2 \boldsymbol{\omega} \cdot \nabla \times\left(\frac{1}{\rho} \nabla \cdot \overline{\bar{\sigma}}\right)
\end{gathered}
$$

where the first term in the right hand side represents the stretching/tilting effect, the second term embodies the dilatation effect, the third term is the baroclinic influence on vorticity and the last term is due to viscous effects. Because according to the $\operatorname{EOS} p\left(v, T, X_{\alpha}\right)$, where $v=m / \rho$ is the molar volume,

$$
\nabla p=\frac{\delta p}{\delta v}\left(-\frac{m}{\rho^{2}}\right) \nabla \rho+\frac{\delta p}{\delta T} \nabla T+\sum_{\alpha=1}^{N} \frac{\delta p}{\delta X_{\alpha}} \nabla X_{\alpha}
$$

where $N$ is the total number of species and $\delta$ denotes a functional derivative, it is clear that the baroclinic term expresses the misalignment of $\nabla \rho$ with $\nabla T$ 
and/or with $\nabla X_{\alpha}$ because

$\nabla\left(\frac{1}{\rho}\right) \times \nabla p=-\frac{1}{\rho^{2}} \nabla \rho \times \nabla p=-\frac{1}{\rho^{2}}\left[\frac{\delta p}{\delta T}(\nabla \rho \times \nabla T)+\sum_{\alpha=1}^{N} \frac{\delta p}{\delta X_{\alpha}}\left(\nabla \rho \times \nabla X_{\alpha}\right)\right]$

Equations (5) and (8) indicate that the composition of a fluid can affect its vortical features, coupling dynamics and thermodynamics. Since the simulations are initiated with error function mean profiles linking the specified different values of $T$ and $X_{\alpha}$ in the upper and lower stream [1,34], the early development of $\nabla T$ and $\nabla X_{\alpha}$ is substantially the result of molecular transport processes. Further in the development of the layer, the baroclinic term represents the effect of molecular transport combined with convective transport. Homogeneous $\left(x_{1}, x_{3}\right)$ plane averages of the above-mentioned four contributions to $\omega_{3}$ are very noisy due to the presence of the HDGM regions and therefore only the activities of these contributions are illustrated in Fig. 1; the "activity" is here defined as the magnitude of the r.m.s.. The homogeneous $\left(x_{1}, x_{3}\right)$ plane average contributions to the enstrophy are displayed in Fig. 2; the corresponding activities (i.e. r.m.s.) are not shown since they parallel those of $\omega_{3}$. Figures 1 and 2 exhibit results at both $t_{t r}^{*}$ and $t_{p p}^{*}$.

In Fig. 1, the r.m.s. of the sources in Eq. (5) shows that at $t_{t r}^{*}$ the stretching/tilting and viscous terms are most active and matching in activity, as these are indeed the phenomena crucial in production and destruction of turbulence; the baroclinic term is a distant third effect, and smallest in magnitude is the dilatation effect. This ranking in importance remains valid at $t_{p p}^{*}$ for R1000p60 and R2000p60. However, for R1000p80 where the fluid is denser and more difficult to distort (i.e. to stretch/tilt), and for R1000p60a in which the vortical features are not so strong (reduced stretching/tilting due to the lack of uphill diffusion), the viscous and baroclinic effects dominate, while the stretching/tilting effect is a close third, followed by the dilatation.

The average of each of the source-term contributions in the enstrophy equation (see Fig. 2) shows that stretching/tilting and baroclinic effects produce enstrophy whereas viscous and dilatation effects destroy enstrophy. At $t_{t r}^{*}$ all these contributions decrease with increasing $p_{0}$ (compare Figs. 2a and 2c), diminish with reduced energy release (compare Figs. 2a and 2e) and increase with $\mathrm{Re}_{0}$ (compare Figs. $2 \mathrm{a}$ and $2 \mathrm{~g}$ ). All these contributions decrease substantially by $t_{p p}^{*}$. The baroclinic effect assumes increased importance at higher $p_{0}$ and larger values of $\nabla \rho$ and $\nabla T$ and/or $\nabla X_{\alpha}$ induced by the larger reaction rates for R1000p60a at $t_{p p}^{*}$, as discussed above.

The insights obtained from this examination of vortical aspects before and after combustion indicate that the HDGM regions play a crucial role in turbulent mixing and turbulent combustion. Atmospheric- $p$ turbulent reaction rate models rely on the $\mathrm{PDF}$ of the scalar dissipation which they use as a 
diffusion coefficient in the $\varsigma$ equation to engage these HDGM effects into the turbulent reaction rate model. The diffusion rate being slower at high $p$, the HDGM effects become more prominent with increasing $p$ and thus will play an increasingly more important role in the modeling of the turbulent reaction rate at high- $p$ conditions.

\subsection{Examination of assumptions used in atmospheric-p turbulent reaction rate models}

The interest here is to examine some of the assumptions representing the backbone of flamelet-type models used in LES. This examination is not an $a$ priori analysis in which SGS models would be tested and their performance compared to the filtered-and-coarsened (FC) DNS database; this analysis and testing are lengthy procedures outside the scope of this study. The goal of the present examination which uses the DNS rather than the FC DNS database, is to evaluate some aspects which might require further in-depth studies for developing high- $p$ turbulent reaction-rate models; in computing all statistics below, the analysis concepts discussed in the introduction of Section 5 are adopted.

\subsubsection{Mixture fraction}

When flames occur in relatively thin regions compared to the Kolmogorov scale, for atmospheric- $p$ combustion it is postulated that turbulence moves the flame and distorts it as an entity rather then penetrating into it and affecting there the reaction. Thus, the interior of the flame is similar to a laminar flame, and turbulence modeling is only necessary for the flow external to the flame. Finding the evolution of the flame is then reduced to obtaining its statistical position. Since previous results [1] indicate that the highest reaction rate is in the diffusion flame, that the diffusion flame region is not visually excessively thick and that the larger reaction rates are found in the regions of larger $|\nabla \rho|$ values, it is reasonable to investigate the veracity, under the present conditions, of the pivotal assumption of the flamelet model that the differential equation satisfied by $\varsigma$ is that of a self-contained conserved scalar, i.e. Eq. (1).

In the present case, for convenience, the definition of $\varsigma$ is based on the fuel mass fraction; mathematically, this definition is entirely consistent with the $\varsigma$ definition based on elements as for this study the only pertinent information is that the fluxes of all species must add to zero. For the single-step reaction of Eq. (2), one can define the ratio $v$

$$
v \equiv \frac{v_{\mathrm{O}_{2}} m_{\mathrm{O}_{2}}}{v_{\mathrm{C}_{7} \mathrm{H}_{16}} m_{\mathrm{C}_{7} \mathrm{H}_{16}}}
$$


where $v_{\mathrm{C}_{7} \mathrm{H}_{16}}$ and $v_{\mathrm{O}_{2}}$ are the stoichiometric coefficients in Eq. (2) and for the present study $\varsigma$ is represented by

$$
\xi \equiv \frac{v Y_{\mathrm{C}_{7} \mathrm{H}_{16}}-Y_{\mathrm{O}_{2}}+Y_{\mathrm{O}_{2}, U}}{v Y_{\mathrm{C}_{7} \mathrm{H}_{16}, L}+Y_{\mathrm{O}_{2}, U}}
$$

with $\xi=0$ in the upper free-stream and $\xi=1$ in the lower free-stream. Using the conservation equations for $\left(\rho Y_{\mathrm{C}_{7} \mathrm{H}_{16}}\right)$ and $\left(\rho Y_{\mathrm{O}_{2}}\right)$, to derive the equation for $\xi$, one obtains

$$
\frac{\partial(\rho \xi)}{\partial t}+\nabla \cdot(\rho \mathbf{u} \xi)=-\frac{1}{v Y_{\mathrm{C}_{7} \mathrm{H}_{16}, L}+Y_{\mathrm{O}_{2}, U}} \nabla \cdot\left(v \mathbf{J}_{\mathrm{C}_{7} \mathrm{H}_{16}}-\mathbf{J}_{\mathrm{O}_{2}}\right)
$$

where $\mathbf{J}_{\alpha}$ is the species $\alpha$ mass flux containing fully multicomponent mass diffusion and the Soret effect (see [1] for details). To transform Eq. (11) to resemble Eq. (1), the quantity $\nabla \cdot\left(\left(\lambda / C_{p}\right) \nabla \xi\right)$ is added and subtracted, where $\lambda$ is computed according to the method presented in Appendix 1 of [1] and $C_{p}$ is computed from the EOS. This results in the following equation

$$
\frac{\partial(\rho \xi)}{\partial t}+\nabla \cdot(\rho \mathbf{u} \xi)=\underbrace{\nabla \cdot\left(\frac{\lambda}{C_{p}} \nabla \xi\right)}_{\text {traditional diffusion }}-\underbrace{\nabla \cdot\left(\frac{\lambda}{C_{p}} \nabla \xi+\frac{v \mathbf{J}_{\mathrm{C}_{7} \mathrm{H}_{16}}-\mathbf{J}_{\mathrm{O}_{2}}}{v Y_{\mathrm{C}_{7} \mathrm{H}_{16}, L}+Y_{\mathrm{O}_{2}, U}}\right)}_{\text {correction to traditional diffusion }}
$$

where the first term in the right hand side represents traditional diffusion under the assumption of unity Lewis number (according to [34], under the present conditions there are five species-specific effective Lewis numbers which are spatially dependent and time dependent, and show substantial departures from unity). The correction term in Eq. (12) contains other dependent variables than $\xi$ (i.e. $p, T$ and $Y_{\alpha}$ ) and thus it differs from the diffusion term in Eq. (1); although Eq. (12) is still of diffusion type, to be consistent with the terminology adopted by others, the correction terms are denoted as a 'source' and represent the fact that Eq. (12) is not a self-contained conservation equation since the knowledge of all thermodynamic variables is necessary to solve for $\xi$.

Examination of the homogeneous plane $\left(x_{1}, x_{3}\right)$ averages of the source terms in Eq. (12) at $t_{p p}^{*}$ (not shown) indicates that the terms are very noisy; nevertheless, these plots indicate that the correction term in Eq. (12) is larger by a factor of 2-3 than the traditional diffusion term. The activity of the source terms in Eq. (12) is illustrated at $t_{p p}^{*}$ in Fig. 3 for the four simulations listed in Table 1. Independent of the case under consideration, the results show that the traditional diffusion term has a similar qualitative variation to the correction term, including structural aspects as a function of $x_{2} / \delta_{\omega, 0}$, but that it highly underestimates the actual diffusion since the correction term has typically double the magnitude of the traditional diffusion term. This commonality of the results for all simulation suggest that effectively, the correction term may be considered as a highly active SGS term in Eq. (12). 
In turbulent non-premixed reactive flows, $\xi$ is typically represented by a $\beta$ PDF [50]; other PDFs can be used if there is physical support for their assumed shape. The basis of the $\beta$ PDF assumption is presented in the study of [51] that was devoted to turbulent mixing. Since the $\beta$ PDF is entirely determined by its mean and variance, these moments were computed from the exact $\xi$ PDF obtained using the DNS database, and then these moments were used to construct what would be considered the most accurate $\beta$ PDF to represent $\xi$ (whereas in typical LES calculations the exact moments are unknown; typically, the mean is obtained from the LES solution and the variance is computed from a model). Using the standard deviation, $\sigma_{D}=\sqrt{\left\langle(\varphi-\langle\varphi\rangle)^{2}\right\rangle}$, of the exact $\xi$ PDF extracted from the DNS, the skewness, $\mathcal{M}_{3}=<(\varphi-<\varphi>$ )$^{3}>/ \sigma_{D}^{3}$, and kurtosis, $\mathcal{M}_{4}=<(\varphi-<\varphi>)^{4}>/ \sigma_{D}^{4}$ were computed. $\mathcal{M}_{3}$ and $\mathcal{M}_{4}$ of the $\beta$ PDF constructed with the exact moments were also computed. The results are displayed in Fig. 4 at $t_{p p}^{*}$ for the simulations listed in Table 1. For all $\mathrm{Re}_{0}=1000$ simulations, the exact PDF skewness and kurtosis approach those of the $\beta \mathrm{PDF}$ in the middle of the layer where the species are most mixed, although substantial departures can be seen at the fuel edge of the layer where mixing still occurs. Clearly, the humps of the skewness and the valleys of the kurtosis on the fuel side, where multi-species mixing is vigorous are similar for all DNS and may be considered as a representative behavior; this behavior cannot be reproduced by the $\beta$ PDF. For R1000p60a, the humps on the fuel side are reduced compared to the other $\mathrm{Re}_{0}=1000$ simulations because there is a smaller number of species mixing at that location, but the departure from the $\beta \mathrm{PDF}$ in the middle of the layer is larger because the $\mathrm{CO}_{2}$ and $\mathrm{H}_{2} \mathrm{O}$ produced in the reaction zone are diffusing into that region devoid of reaction products, whereas for R1000p60 and R1000p80 those species already exist in the layer. For the R2000p60 case, neither in the middle of the layer nor on the fuel edge of the layer do the skewness and kurtosis of the exact $\mathrm{PDF}$ approach the $\beta \mathrm{PDF}$; this is because mixing is still intense in that region (see [1]). However, the exact PDF skewness and kurtosis approach those of the $\beta \mathrm{PDF}$ at the upper part of the mixing layer where burning occurs (see [1]) and this observation holds for all cases. The common trends to all simulations suggest that the skewness and kurtosis of the exact PDF will not be well reproduced in the mixing region (i.e. here $x_{2} / \delta_{\omega, 0} \in[-5,5]$ ) by the $\beta \mathrm{PDF}$ computed with the exact moments.

Since coincidence of the moments does not signify coincidence of the PDFs, the exact PDFs in homogeneous $\left(x_{1}, x_{3}\right)$ planes and the corresponding $\beta$ PDFs are presented in Fig. 5 for all simulations listed in Table 1. That is, similar to Fig. 4, the interest is here only in comparing the shape of the $\beta \mathrm{PDF}$ with the exact PDF rather than in its quantitative accuracy; the accuracy of the moments used to construct the $\beta \mathrm{PDF}$ is a further issue which is only relevant if the shape of the $\beta \mathrm{PDF}$ is satisfactory. The $x_{2} / \delta_{\omega, 0}$ stations are selected to encompass both mixing and combustion. The $x_{2} / \delta_{\omega, 0}=-4$ station represents 
mixing on the fuel side (see the HDGM regions in [1]) and consistently $\xi$ has a very high probability of being unity and a small probability of $\xi \in[0.5,1]$. The $\beta$ PDF shows larger probability than the exact PDF near $\beta \operatorname{PDF} \xi=1$ and smaller probability elsewhere indicating that it underestimates mixing. The PDFs at station $x_{2} / \delta_{\omega, 0}=0$ portray mixing away from the flame. At $x_{2} / \delta_{\omega, 0}=8$, the PDFs are computed through the flame. Finally, at $x_{2} / \delta_{\omega, 0}=$ 12 one encounters the outer edge of the flame on the oxidizer side for R1000p80 and R1000p60 while this station is still through the flame for R1000p60a and R2000p60. The common feature is that the qualitative aspect of all exact PDFs is not captured by the corresponding $\beta$ PDFs; however, there is better agreement for the cases for which the PDF is computed at closer locations to the outer edge of the flame and more inaccurate prediction for the cases where burning is still present. Considering the commonalities of the comparison between the $\beta \mathrm{PDF}$ and the exact PDF for all DNS, the trends show better success in the flame region and more deficiencies in the mixing region.

\subsubsection{The Conditional Source-term Estimation model}

The Conditional Moment Closure [12,13] (CMC) method stems from the necessity to model the filtered turbulent reaction rate in LES. The method postulates that the reactive species and $\xi$ are correlated, meaning that their fluctuations are correlated as well. The correlation of the fluctuations implies that conditioning the reactive species variables on $\xi$ reduces the problem to modeling quantities having smaller fluctuations about the conditional mean than they would have in physical space, making thus the conditioned reaction rate easier to model; for example, simple first-order closures have been used to model the conditioned reaction rate [14] in atmospheric- $p$ flows. In CMC, the transport equations for the conditional averages are solved as functions of time, space and $\xi$, and this last dimension makes the CMC method very costly to implement. The Conditional Source-term Estimation (CSE) method [16] was developed to remove $\xi$ as a dimension, and proposed to make it unnecessary to solve the conditional average transport equations needed to obtain the solution in CMC; more details on the method can be found in $[16,52,53,54]$ and an interesting variation of CSE using a Doubly Conditional Source-term Estimation in Reynolds Averaged Navier Stokes simulations to compute atmospheric- $p$ turbulent jet flames is provided in [55]. The basic assumption of the CSE model is that the conditional mean reaction rate $\left\langle\dot{\omega}\left(Y_{\alpha}, T\right)\right| \zeta>$ can be written as

$$
<\dot{\omega}\left(Y_{\alpha}, T\right) \mid \zeta>=\dot{\omega}\left(<Y_{\alpha}|\zeta>,<T| \zeta>\right)
$$

where $\alpha$ is $\mathrm{C}_{7} \mathrm{H}_{16}$ and $\mathrm{O}_{2}$, and $\zeta$ represents the sample space of possible $\xi$ values. A more accurate representation of the mean reaction rate is $<$ $\dot{\omega}\left(\rho, Y_{\alpha}, T\right) \mid \zeta>$; however, if the assumption of Eq. (13) is not satisfied, clearly 
$<\dot{\omega}\left(\rho, Y_{\alpha}, T\right) \mid \zeta>=\dot{\omega}\left(<\rho\left|\zeta>,<Y_{\alpha}\right| \zeta>,<T \mid \zeta>\right)$ will not be satisfied either. Thus, the evaluation of Eq. (13) is the simplest that can be performed for the CSE model. According to the analysis method explained in the introduction of Section 5 , for each DNS the sample space is that obtained at $t_{p p}^{*}$.

One requirement in using the CSE method is that a good approximation for the PDF of $\xi$ is necessary to obtain reaction rate accuracy. This point is clearly illustrated in Fig. 6 where the unconditioned homogeneous $\left(x_{1}, x_{3}\right)$ planar averages, i.e. $<\dot{\omega}\left(\rho, Y_{\alpha}, T\right) \mid \zeta>$, are shown as calculated from the DNS, and also as computed according to CSE either using the exact $\xi$ PDF or the $\beta$ PDF in which the exact moments extracted from the DNS are used. The results verify that the reaction rate modeled by the CSE computed with the exact $\xi$ PDF is indeed very close to the reaction rate extracted from the DNS and it also shows that in the region of maximum reaction the CSEmodeled turbulent reaction rate calculated using the $\beta$ PDF computed with the accurate moments, deviates from the exact reaction rate; since the results for the four DNS are similar, we conjecture that this finding would hold if the PDF were computed over an ensemble of DNS solutions.

To evaluate the assumption of Eq. (13), the conditional reaction rate is displayed in Fig. 7 at the same stations $x_{2} / \delta_{\omega, 0}=-4,0,8$ and 12 as $\xi$ was shown in Fig. 5. Comparisons are made between the conditional reaction rate computed from DNS, i.e. the left hand side of Eq. (13), and the reaction rate computed with the conditional moments, i.e. the right hand side of Eq. (13). The performance of the CSE model assumption is quite erratic away from intense combustion $\left(x_{2} / \delta_{\omega, 0}=-4\right.$ and 0$)$, i.e. in regions of minimal reaction rate (e.g. precursors to ignition) in which reaction-rate fluctuations are small but the fluctuations of $\xi$ may be large due to intense turbulent mixing, thereby invalidating the assumption of the correlation between the fluctuations of these quantities. Also, in these regions of relatively minimal reaction rate, $T$ is relatively small making the fluid dense and real-gas effects important. Since the PR EOS is a very non-linear relationship among $\left(p, Y_{\alpha}, T, v_{P R}\right)$ where $v_{P R}$ is the PR molar volume, it is doubtful whether the decoupling of $Y_{\alpha}$ and $T$ according to Eq. (13) is valid. Previous studies in the context of atmospheric- $p$ non-reacting flows [56] in which the perfect gas EOS was used have shown that although for single-phase flows $T$ and $Y_{\alpha}$ were not correlated at the DNS, LES and SGS scales, in two-phase flows with evaporation $T$ and $Y_{\alpha}$ were correlated at all three scales and the difference between single-phase and two-phase flow results was attributed to the role of evaporation, i.e. a thermodynamic process. Thus, the erratic performance of the CSE model in the regions of minimal reaction rate, including ignition, can be attributed to the combined effects of lack of correlation between reaction-rate fluctuations and $\xi$ fluctuations, and to strong real-gas effects which correlate the thermodynamic variables.

However, in the intense reaction regime $\left(x_{2} / \delta_{\omega, 0}=8\right.$ and 12$)$, the CSE model is 
consistently excellent in regions containing substantial amounts of $\mathrm{O}_{2}$ (small $\xi$ ) where the reaction peaks, and slightly deviates from the DNS results in regions containing more $\mathrm{C}_{7} \mathrm{H}_{16}$ (large $\xi$ ). The conjecture is that the success of CSE in these high- $T$ regions is due to the fluid mixture being closer to a perfect gas and to the assumption of the correlation between species mass fraction fluctuations and $\xi$ fluctuation being better satisfied. These common trends suggest is that if (1) a high-fidelity $\xi$ PDF can be obtained to make the CSE model accurate in the high reaction-rate regions, and (2) additional modeling could mitigate the limitations of the CSE model in the small reaction-rate regime, then the CSE model would be useful for modeling high- $p$ turbulent reaction rates.

\section{Summary and conclusions}

To understand the modeling needs and the applicability of selected atmospheric$p$-developed turbulent reaction-rate models to high-pressure (high- $p$ ) conditions, an investigation is undertaken to examine the turbulent evolution of high- $p$ turbulent reactive flows and assess assumptions of these selected models. The information necessary for the study is provided by a previously created Direct Numerical Simulation (DNS) database of a temporal mixing layer composed of two counterflowing streams of different initial compositions. The governing equations include a fully multicomponent species mixing model, a real-gas equation of state (EOS) and a generic single-step reaction involving five species. The DNS realizations represent turbulent mixing and reaction initiation in a turbulent high- $p$ environment, achieved at a realization-specific non-dimensional time $t_{t r}^{*}$, for different initial Reynolds numbers, various initial pressures and several initial composition of the two streams. The simulations are conducted past the non-dimensional time $t_{p p}^{*}$ at which the volumeaveraged $p$ reached a maximum. Details of the flow and flame have been discussed elsewhere [1]; of interest to the present study is the occurrence of high density-gradient magnitude (HDGM) regions and the fact that the flame is of partially-premixed type at $t_{p p}^{*}$, with the highest reaction rates occurring in the diffusion flame located in HDGM regions. These flame characteristics indicated that mixture-fraction-based models should be selected for examination as potential turbulent reaction-rate models. Since the flow is unsteady and non-ergodic, the flames are primarily examined at $t_{p p}^{*}$ and common aspects of behavior are considered representative of the high- $p$ aspects.

A detailed examination of the vorticity and enstrophy equations at $t_{t r}^{*}$ and $t_{p p}^{*}$ using the database led to the conclusion that the baroclinic effect becomes increasingly important for reacting situations when compared to mixing alone, and also (consistently) as the reaction is more vigorous; this effect was attributed to the fact that the baroclinic term is a measure of the misalignment 
between the density gradient and the temperature gradient and/or between the density gradient and the mole fraction gradients, all of which increase when combustion occurs. Considering the finding that vorticity was created by the HDGM regions, it is clear that the HDGM aspect must be embedded into any successful turbulent reaction rate model.

On examining the mixture-fraction equation, it was found that the r.m.s. magnitude of differential diffusion dominates that of the mixture fraction diffusion by approximately a factor of two. However, the local qualitative behavior of these two terms is similar, indicating that perhaps a subgrid-scale modeling could be used in the future to restore to the mixture fraction equation its self-contained form in which it only depends on the mixture fraction. Since in non-premixed turbulent reactive flows the mixture fraction PDF is typically represented by the $\beta \mathrm{PDF}$, a $\beta$ PDF was constructed for each simulation using the exact moments extracted from the DNS solution. The findings show that the $\beta \mathrm{PDF}$ does not reproduce well the shape of the mixture fraction PDF computed from the corresponding DNS solution. A variant of the Conditional Moment Closure, the Conditional Source-term Estimation (CSE) model, in which the mixture fraction still plays an important role in that the accuracy of the model crucially depends on the accuracy of the utilized mixture fraction PDF, was also examined from the point of common features of all DNS. Planar averages of the reaction rate show that when the DNS-extracted mixture fraction PDF was used, the CSE shows excellent performance in reproducing the reaction rate, but the performance deteriorates when the $\beta$ PDF constructed with the exact moments is used. The evaluation of the CSE model assumption reveals substantial departures in regions of minimal reaction rate where the reaction-rate fluctuations and mixture-fraction fluctuations do not correlate well and in which the thermodynamic variables are strongly coupled through the real-gas EOS. However, in regions of intense reaction the comparison is very favorable particularly in regions of peak reaction rate. This means that ignition events may be modeled problematically using CSE, but that if an accurate $\xi$ PDF can be obtained (e.g. using subgrid-scale modeling of the differential diffusion effects in the mixture-fraction equation) then the CSE model could be rendered accurate in the high reaction-rate regions. Assuming that these models could be revised for increased accuracy, the overwhelming remaining issue is their utilization in turbulent reactive-flow simulations containing multi-reaction chemistry.

\section{Acknowledgments}

This work was conducted at the Jet Propulsion Laboratory (JPL) of the California Institute of Technology (Caltech) and sponsored by the Department of Energy (DOE), Basic Energy Sciences (BES) under the direction of Drs. 
Wade Sisk and Mark Pendersen. The contributions of Dr. Giulio Borghesi and Dr. Kenneth G. Harstad are acknowledged. The computational resources were provided by the NASA Advanced Supercomputing at Ames Research Center under the Aeronautics Research Mission Directorate program (Dr. Michael Rogers) and by National Energy Research Supercomputing Center of the Department of Energy.

\section{References}

[1] J. Bellan, Direct Numerical Simulation of a high-pressure turbulent reacting temporal mixing layer, Combust. Flame, accepted for publication (2016)

[2] V. Sankaran, C. Merkle, Fundamental physics and model assumptions in turbulent combustion models for aerospace propulsion, 50th AIAA/ASME/SAE/ASEE Joint Propulsion Conference, Cleveland, OH, 2014.

[3] S.B. Pope, PDF methods for turbulent reactive flows, Progr. Energy Combust. Sci., 11(2) (1985) 119-192.

[4] S. Srinivasan, R. Ranjan, S. Menon, Flame dynamics during combustion instability in a high-pressure, shear-coaxial injector combustor, Flow Turbulence Combust 94 (2015) 237-262.

[5] R.W. Bilger, The structure of diffusion flames, Comb. Sci. Techn., 13 (1976) 155-170.

[6] R.W. Bilger, S.H. Stårner, R.J. Kee, On reduced mechanisms for methane/air combustion in nonpremixed flames, Combust. Flame 80 (1990) 135-149

[7] H. Pitsch, N. Peters, A consistent flamelet formulation for non-premixed combustion considering differential diffusion effects, Combust. Flame, 114 (1998) 26-40.

[8] N. Peters, Turbulent Combustion, Cambridge University Press, 2000

[9] R. Krishna, Uphill diffusion in multicomponent mixtures, Chemical Society Reviews, 44(10) (2015) 2812-2836.

[10] A.R. Kerstein, One-dimensional turbulence: model formulation and application to homogeneous turbulence, shear flows and buoyancy stratified flows, J. Fluid Mech. 392 (1999) 277-334.

[11] J.C. Sutherland, P.J. Smith, and J.H. Chen, Quantification of differential diffusion in nonpremixed systems, Combust. Theory Modelling, 9(2) (2005) 365-383.

[12] A.Yu. Klimenko, Multicomponent diffusion of various admixtures in turbulent flow, Fluid Dynamics 25 (1990) 327-334. 
[13] R.W. Bilger, Conditional moment methods for turbulent reactive flow, Phys. Fluids. 5 (1993) 436-444.

[14] A. Kronenburg, E. Mastorakos, The conditional moment closure model, Turbulent Combustion Modeling. Eds. (Echekki, T. and Mastorakos, E.), Fluid Mechanics and its Applications 95, Springer Science, Chapter 5 (2011) 91-117.

[15] W.K. Bushe, H. Steiner, Conditional moment closure for large eddy simulation of nonpremixed turbulent reacting flows, Phys. Fluids, 11(7) (1999) 1896-1906

[16] H. Steiner, W.K. Bushe, Large Eddy Simulation of a turbulent reacting jet with Conditional Source-term Estimation, Phys. Fluids 13(3) (2001) 754-769.

[17] T. Poinsot, D. Veynante, Theoretical and Numerical Combustion, Second Edition 3rd Edition, CNRS, 2011

[18] R.S. Barlow, M.J. Dunn, M.S. Sweeney, S. Hochgreb, Effects of preferential transport in turbulent bluff-body-stabilized lean premixed $\mathrm{CH} 4 /$ air flames, Combust. Flame 159 (2012) 2563-2575

[19] R.W. Bilger, Molecular transport effects in turbulent diffusion flames at moderate Reynolds number, AIAA J., 20(7) (1982), 962-970.

[20] W. L. Chan, A Higher-Order Flamelet Model for Turbulent Combustion Simulations, $\mathrm{PhD}$ thesis, University of Michigan, 2016

[21] A. Scholtissek, W.L. Chan, H. Xu, F. Hunger, H. Kolla, J.H., Chen, M. Ihme, C. Hasse, A multi-scale asymptotic scaling and regime analysis of flamelet equations including tangential diffusion effects for laminar and turbulent flames, Combust. Flame, 162(4) (2015) 1507-1529.

[22] M. J. Cleary, A. Y. Klimenko, Multiple Mapping Conditioning: A new modelling framework for turbulent combustion, Turbulent Combustion Modeling, Fluid Mechanics and its Applications 95, Eds. T. Echekki and E. Mastorakos, Springer Science+Business Media B. V.,143-173, 2011

[23] J. Keizer, Statistical Thermodynamics of Nonequilibrium Processes,SpringlerVerlag, New York, 1987.

[24] K. Harstad, J. Bellan, Mixing rules for multicomponent mixture mass diffusion coefficients and thermal diffusion factors, J. Chem. Phys. 120(12) (2004) 56645673.

[25] K. Harstad, R.S. Miller, J. Bellan, Efficient high pressure state equations, AIChE J. 43 (1997) 1605-1610.

[26] E. Mastorakos, T.A. Baritaud, T.J. Poinsot, Numerical simulations of autoignition in turbulent mixing flows, Combust. Flame 109 (1997) 198-223.

[27] S. Sreedhara, K.N. Lakshmisha, 2000 Direct numerical simulation of autoignition in a non-premixed, turbulent medium, Proc. Combust. Inst. 28(1) (2000) 25-33. 
[28] G. Borghesi, E. Mastorakos, R.S. Cant, Complex chemistry DNS of n-heptane spray autoignition at high pressure and intermediate temperature conditions, Combust. Flame 160 (2013) 1254-1275.

[29] C. Westbrook, F.L. Dryer, Simplified reaction mechanisms for the oxidation of hydrocarbon fuels in flames, Combust. Sci. Techn. 27 (1981) 31-43.

[30] M R. Overholt, S.B. Pope, Direct numerical simulation of a statistically stationary, turbulent reacting flow, Combust. Theory Modelling 3 (1999) 371408.

[31] M. Mortensen , S.M. de Bruyn Kops, C.M. Cha, Direct numerical simulations of the double scalar mixing layer Part II: Reactive scalars, Combust. Flame 149 (2007) 392-408.

[32] I.L. Pioro, H.F. Khartabil, R.B. Duffey, Heat transfer to supercritical fluids flowing in channels - empirical correlations (survey), Nuclear Engineering and Design 230(1-3) (2004) 69-91.

[33] J.Y Yoo, The turbulent flows of supercritical fluids with heat transfer, Annu. Rev. Fluid Mech. 45 (2013) 495-525.

[34] E. Masi, J. Bellan, K. Harstad, N. Okong'o, Multi-species turbulent mixing under supercritical-pressure conditions: modeling, Direct Numerical Simulation and analysis revealing species spinodal decomposition, J. Fluid Mech. 721 (2013) 578-626.

[35] G. Borghesi, J. Bellan, Irreversible entropy production rate in high-pressure turbulent reactive flows, Proc. of the Comb. Inst., 35 (2015) 1537-1547.

[36] A.I Olemskoŭ, E.A. Toropov, I.A. Sklyar, Self-consistent theory of the transition of an unstable thermodynamic system from spinodal to heterophase kinetics, Sov. Phys. JETP 73(3) (1991) 545-551.

[37] R.W. Balluffi, S.M. Allen, W.C. Carter, Kinetics of Materials, John Wiley \& Sons, Inc., 2005.

[38] M. Oschwald, A. Schik, Supercritical nitrogen free jet investigated by spontaneous Raman scattering, Exp. Fluids. 27 (1999) 497-506.

[39] B. Chehroudi, D. Talley, E. Coy, Visual characteristics and initial growth rates of round cryogenic jets at subcritical and supercritical pressures, Phys. Fluids. 14(2) (2002) 850-861.

[40] C. Segal, S.A. Polikhov, Subcritical to supercritical mixing, Phys. Fluids 20 (2008) 052101.

[41] Z. Falgout, M. Rahm, D. Sedarsky, M. Linne, Gas/fuel jet interfaces under high pressures and temperatures, Fuel, 168 (2016) 14-21.

[42] Z. Falgout, M. Rahm, Z. Wang, M. Linne, Evidence for supercritical mixing layers in the ECN Spray A, Proc. Comb. Inst., 35 (2015) 1579-1586. 
[43] http://www.sandia.gov/ecn/cvdata/targetCondition/sprayA.php.

[44] I.A. Hannoun, H.J.S. Fernando, E.J. List, Turbulence structure near a sharp density interface, J. Fluid Mech. 189 (1998) 189-209.

[45] N. Okong'o, J. Bellan, Direct numerical simulation of a transitional supercritical binary mixing layer: heptane and nitrogen, J. Fluid Mech. 464 (2002) 1-34.

[46] N. Okong'o, K. Harstad, J. Bellan, Direct numerical simulations of $\mathrm{O}_{2} / \mathrm{H}_{2}$ temporal mixing layers under supercritical conditions, AIAA J. 40(5) (2002) 914-926.07) 392-408.

[47] E.B Nauman, D.Q. He, Nonlinear diffusion and phase separation, Chem. Eng. Sci. 56 (2001) 1999-2018.

[48] Y. Zhang, Geochemical kinetics, Princeton University Press, 2008.

[49] A.J. Chorin, Vorticity and turbulence, Applied Mathematical Sciences, 103, Springer Verlag, New York., 1991.

[50] R.O. Fox, Computational models for turbulent reactive flows, Cambridge University Press, 2003

[51] S.S. Girimaji, Assumed $\beta$-pdf model for turbulent mixing: validation and extension to multiple scalar mixing, Comb Sci. Techn. 78 (4-6) (1991) 177-196.

[52] W.K. Bushe, H. Steiner, Laminar flame decomposition for conditional sourceterm estimation, Phys. Fluids 15(6) (2003)1564-1575.

[53] B. Jin, R. Grout, W.K. Bushe, Conditional source-term estimation as a method for chemical closure in premixed turbulent reactive flow, Flow Turbulence Combust. 81 (2008) 563-582.

[54] N. Shahbazian, M.M. Salehi, C.P.T. Groth, Ö. Gülder, W.K. Bushe, Performance of conditional source-term estimation model for LES of turbulent premixed flames in thin reaction zones regime, Proci. Comb. Inst. 35(2) (2015) $1367-1375$.

[55] D. Dovizio, J. W. Labahn, C. B. Devaud, Doubly Conditional Source-term Estimation (DCSE) applied to a series of lifted turbulent jet flames in cold air, Combustion and Flame 162(5) (2015) 1976-1986.

[56] L.C. Selle, J. Bellan, Evaluation of assumed-PDF methods in two-phase flows using direct numerical simulation, Proci. Comb. Inst. 31 (2007) 2273-2281. 


\begin{tabular}{ccccccccccc}
\hline Run & $\operatorname{Re}_{0}$ & $p_{0}$ & $\left(\frac{\rho_{L}}{\rho_{U}}\right)_{0}$ & $L_{2}$ & $N_{1} \times N_{2} \times N_{3}$ & $\Delta x$ & $t_{t r}^{*}$ & $\operatorname{Re}_{m, t r} t_{p p}^{*}$ & $\operatorname{Re}_{m, p p}$ \\
& $(\mathrm{~atm})$ & $(\mathrm{m})$ & $\left(10^{-4} \mathrm{~m}\right)$ & & & \\
\hline R1000p60 & 1000 & 60 & 9.68 & 0.221 & $480 \times 530 \times 288$ & 4.17 & 90 & 1766 & 134 & 2221 \\
R1000p80 & 1000 & 80 & 10.03 & 0.216 & $584 \times 630 \times 344$ & 3.42 & 85 & 1749 & 125 & 2106 \\
R1000p60a 1000 & 60 & 12.56 & 0.221 & $480 \times 530 \times 288$ & 4.17 & 90 & 1762 & 136 & 2218 \\
R2000p60 & 2000 & 60 & 9.68 & 0.209 & $768 \times 804 \times 460$ & 2.60 & 117 & 3804 & 171 & 4106 \\
\hline
\end{tabular}

Table 1

List of the Direct Numerical Simulation realizations and associated resolution. $L_{i}$ is the size of the domain in the $x_{i}$ direction, in meters. For all layers, $L_{1}=0.2 \mathrm{~m}$ and $L_{3}=0.12 \mathrm{~m}$. For all simulations $T_{U}=1000 \mathrm{~K}, T_{L}=600 \mathrm{~K}$. Information regarding the initial upper stream and lower stream compositions is provided in Table 2. The subscripts $t r$ and $p p$ denote the transitional time and pressure-peak time, respectively.

\begin{tabular}{ccccccc}
\hline Run & Stream & $Y_{\mathrm{H}_{2} \mathrm{O}}$ & $Y_{\mathrm{CO}_{2}}$ & $Y_{\mathrm{O}_{2}}$ & $Y_{C_{7} H_{16}}$ & $Y_{N_{2}}$ \\
\hline All simulations & $U$ & 0.01 & 0.035 & 0.20 & 0.00 & 0.755 \\
(except R1000p60a) & $L$ & 0.01 & 0.035 & 0.00 & 0.955 & 0.00 \\
\hline R1000p60a & $U$ & 0.00 & 0.00 & 0.245 & 0.00 & 0.755 \\
& $L$ & 0.00 & 0.00 & 0.00 & 1.00 & 0.00 \\
\hline
\end{tabular}

Table 2

Initial mass fractions of water, carbon dioxide, oxygen, n-heptane and nitrogen in the upper and lower streams.

Table 3

\begin{tabular}{lllllll}
\hline Species & Species & $m_{\alpha}$ & $T_{c}$ & $p_{c}$ & $v_{c}$ & $\Omega$ \\
& label $(\alpha)$ & $(\mathrm{kg} / \mathrm{kmol})$ & $(\mathrm{K})$ & $($ bar $)$ & $\left(\frac{10^{-3} \mathrm{~m}^{3}}{\mathrm{kmol}}\right)$ & \\
\hline $\mathrm{H}_{2} \mathrm{O}$ & 1 & 18.015 & 647.3 & 221 & 57.1 & 0.344 \\
$\mathrm{CO}_{2}$ & 2 & 44.01 & 304.1 & 73.8 & 93.9 & 0.239 \\
$\mathrm{O}_{2}$ & 3 & 32.0 & 154.6 & 50.43 & 73.4 & 0.025 \\
$\mathrm{C}_{7} \mathrm{H}_{16}$ & 4 & 100.2 & 540.2 & 27.4 & 432 & 0.349 \\
$\mathrm{~N}_{2}$ & 5 & 28.013 & 126.26 & 33.4 & 89.8 & 0.039 \\
\hline
\end{tabular}

Species properties. The species are listed in order of index $\alpha$. Subscript $c$ refers to the critical point of the species, $v$ is the molar volume and $\Omega$ is the acentric factor. 

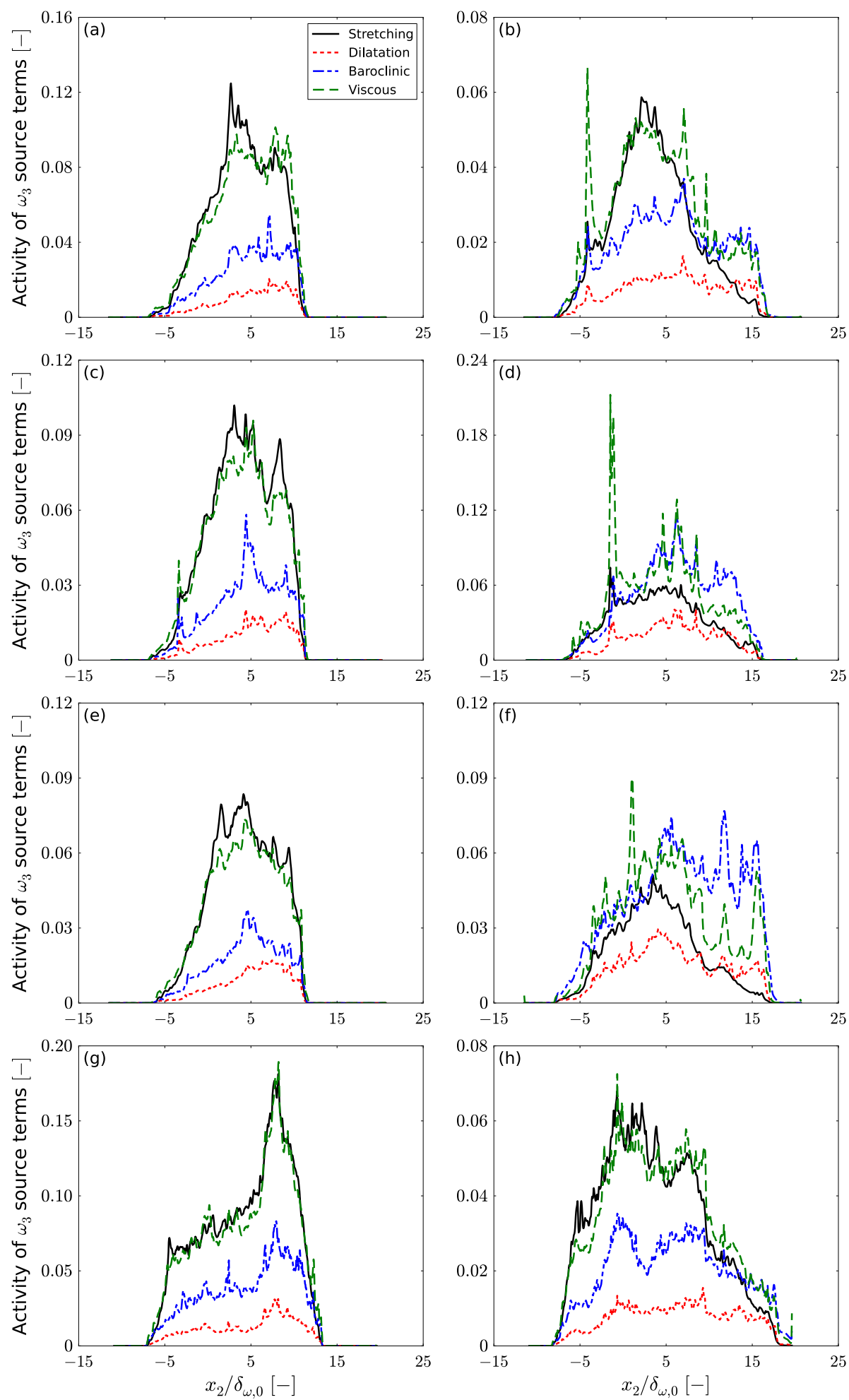

Fig. 1. Homogeneous-plane activity of source terms in the vorticity equation at $(\mathrm{a}, \mathrm{c}, \mathrm{e}, \mathrm{g}) t_{t r}^{*}$ and $(\mathrm{b}, \mathrm{d}, \mathrm{f}, \mathrm{h}) t_{p p}^{*}$ for $(\mathrm{a}, \mathrm{b}) \mathrm{R} 1000 \mathrm{p} 60,(\mathrm{c}, \mathrm{d}) \mathrm{R} 1000 \mathrm{p} 80,(\mathrm{e}, \mathrm{f}) \mathrm{R} 1000 \mathrm{p} 60 \mathrm{a}$, and $(\mathrm{g}, \mathrm{h}) \mathrm{R} 2000 \mathrm{p} 60$. The rms of the source terms have have been non-dimensionalized through dividing by $\left(\Delta U_{0} / \delta_{\omega, 0}\right)^{2}$. 

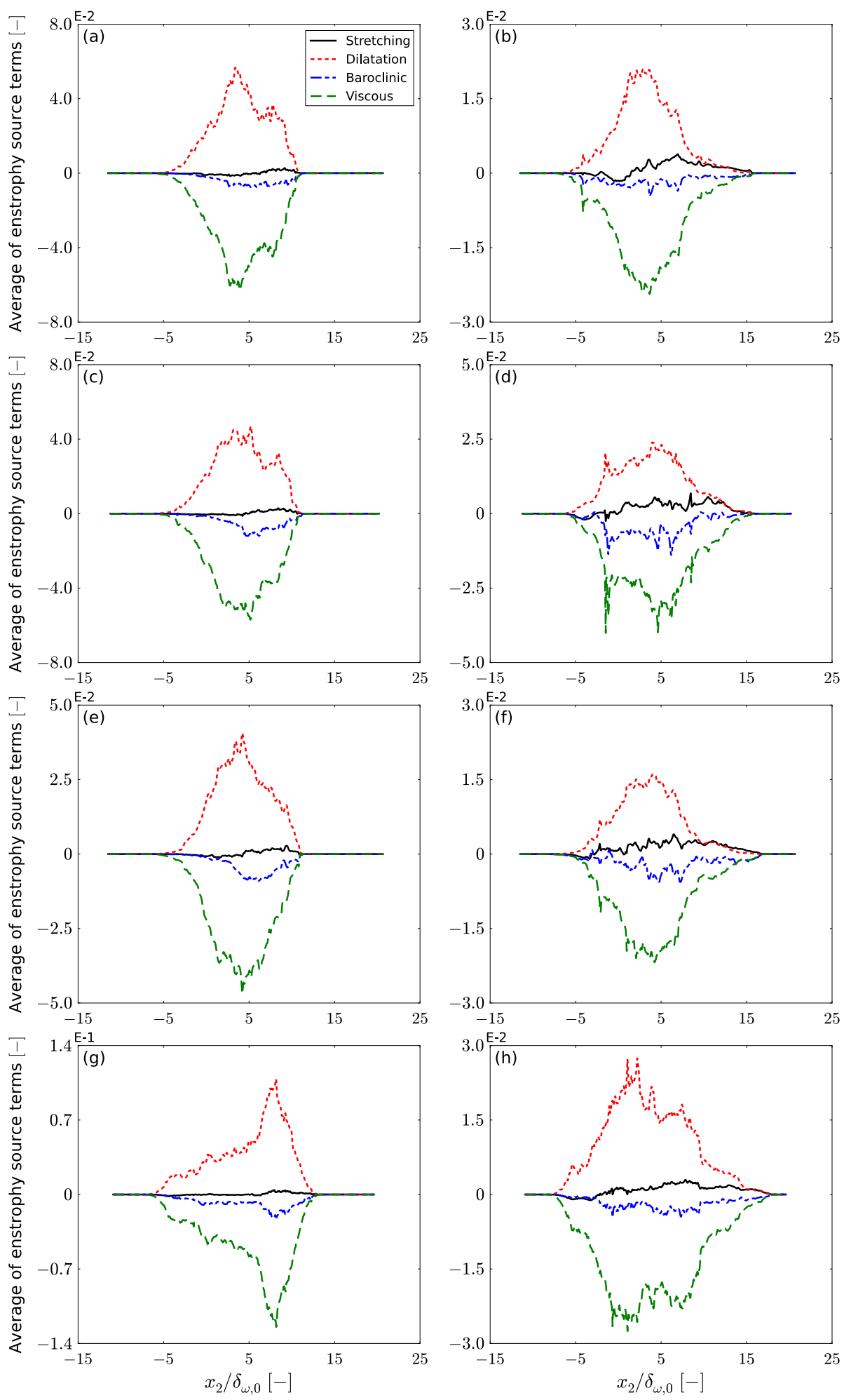

Fig. 2. Homogeneous-plane averages of source terms in the enstrophy equation at $(\mathrm{a}, \mathrm{c}, \mathrm{e}, \mathrm{g}) t_{t r}^{*}$ and $(\mathrm{b}, \mathrm{d}, \mathrm{f}, \mathrm{h}) t_{p p}^{*}$ for $(\mathrm{a}, \mathrm{b}) \mathrm{R} 1000 \mathrm{p} 60,(\mathrm{c}, \mathrm{d}) \mathrm{R} 1000 \mathrm{p} 80,(\mathrm{e}, \mathrm{f}) \mathrm{R} 1000 \mathrm{p} 60 \mathrm{a}$, and $(\mathrm{g}, \mathrm{h}) \mathrm{R} 2000 \mathrm{p} 60$. The averages of the source terms have have been non-dimensionalized through dividing by $\left(\Delta U_{0} / \delta_{\omega, 0}\right)^{3}$. 

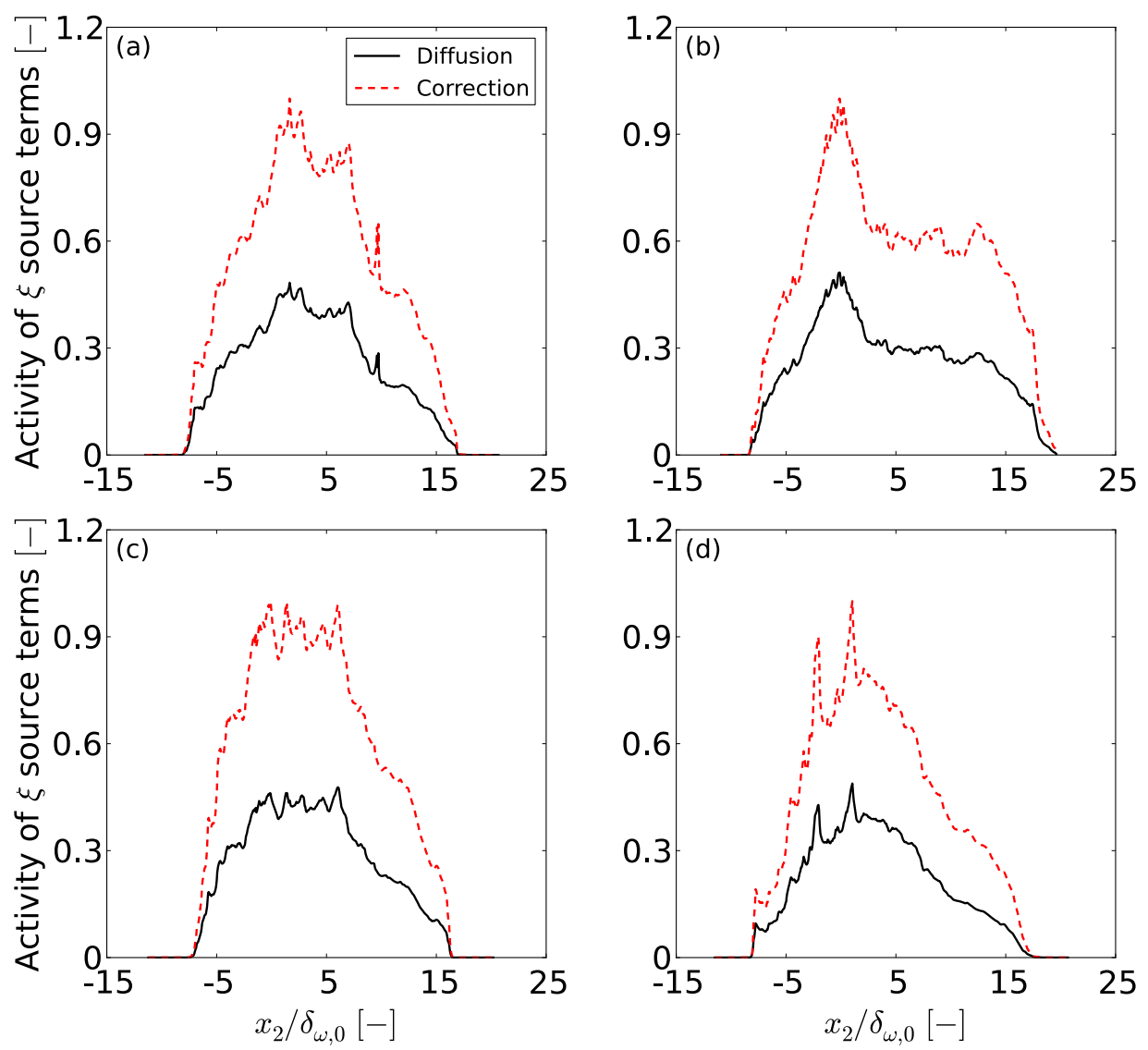

Fig. 3. Activity of the corrrection (here denoted as source) terms in equation (12) for (a) R1000p60, (b) R2000p60, (c) R1000p80 and (d) R1000p60a. All at the respective $t_{p p}^{*}$. The plotted quantities were normalized by $\max _{V}\left(\nabla \cdot \mathbf{J}_{\mathrm{C}_{7} \mathrm{H}_{16}}, \nabla \cdot \mathbf{J}_{\mathrm{O}_{2}}\right)$. 

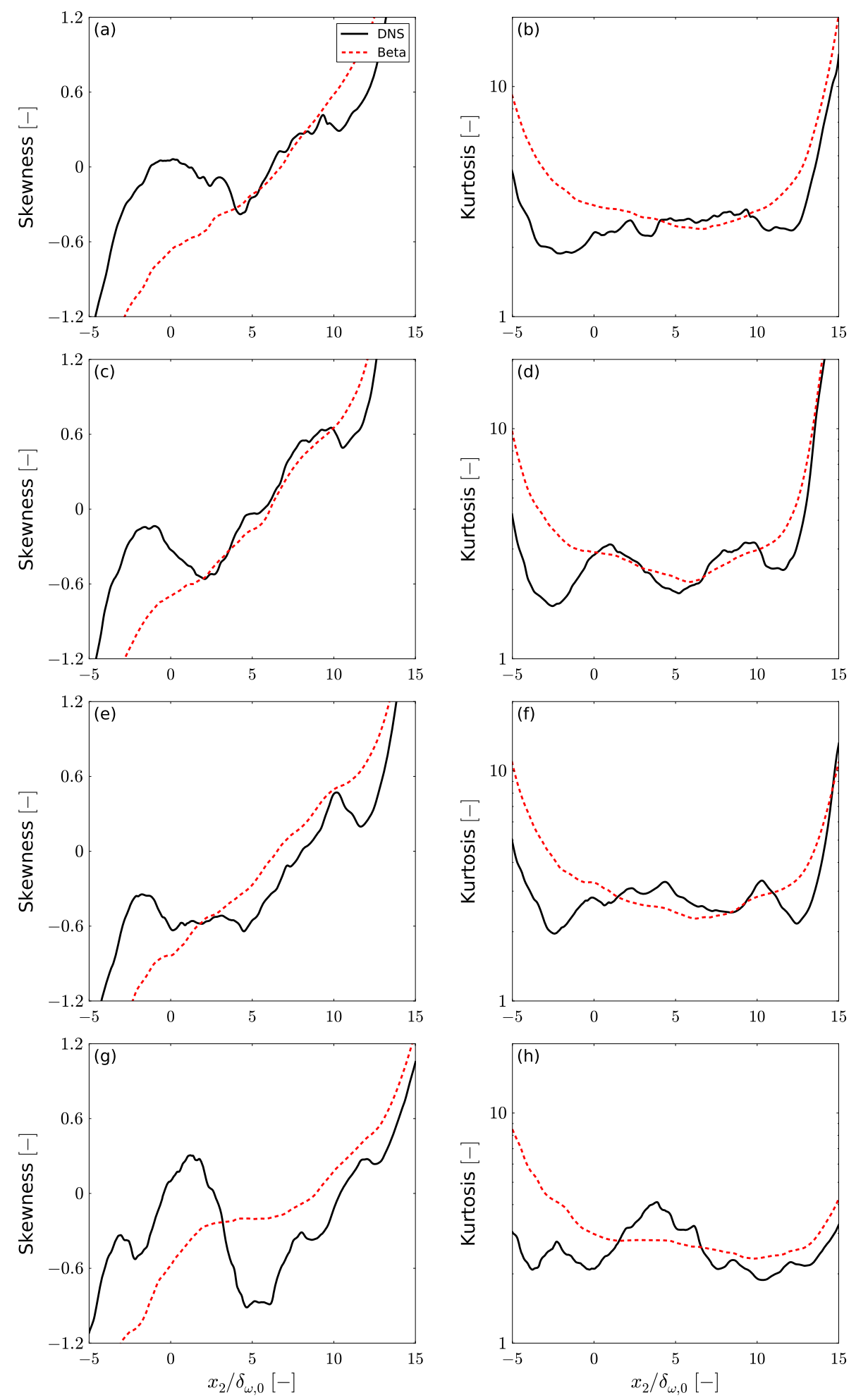

Fig. 4. Planar PDF statistics for $\xi$ at $t_{p p}^{*}$ for (a,b) R1000p60, (c,d) R1000p80, (e,f) R1000p60a, and (g,h) R2000p60. (a,c,e,g) skewness and (b,d,f,h) kurtosis. 

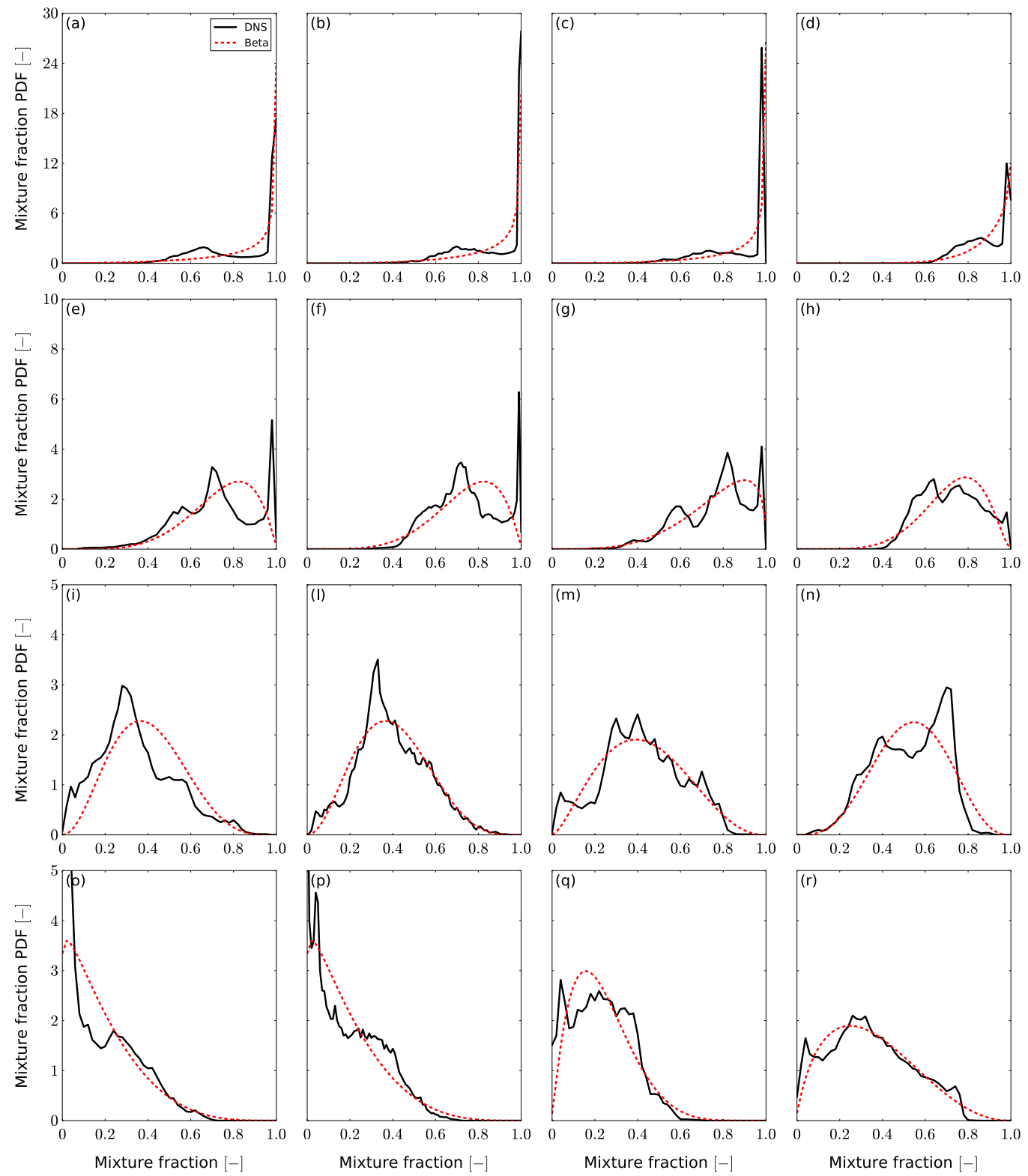

Fig. 5. Mixture fraction probability density functions at different $x_{2} / \delta_{\omega, 0}$ locations, all at the respective $t_{p p}^{*}$. First column, R1000p80; second column, R1000p60; third column, R1000p60a; fourth column, R2000p60. First row, $x_{2} / \delta_{\omega, 0}=-4$; second row, $x_{2} / \delta_{\omega, 0}=0$; third row, $x_{2} / \delta_{\omega, 0}=8$; fourth row, $x_{2} / \delta_{\omega, 0}=12$. 

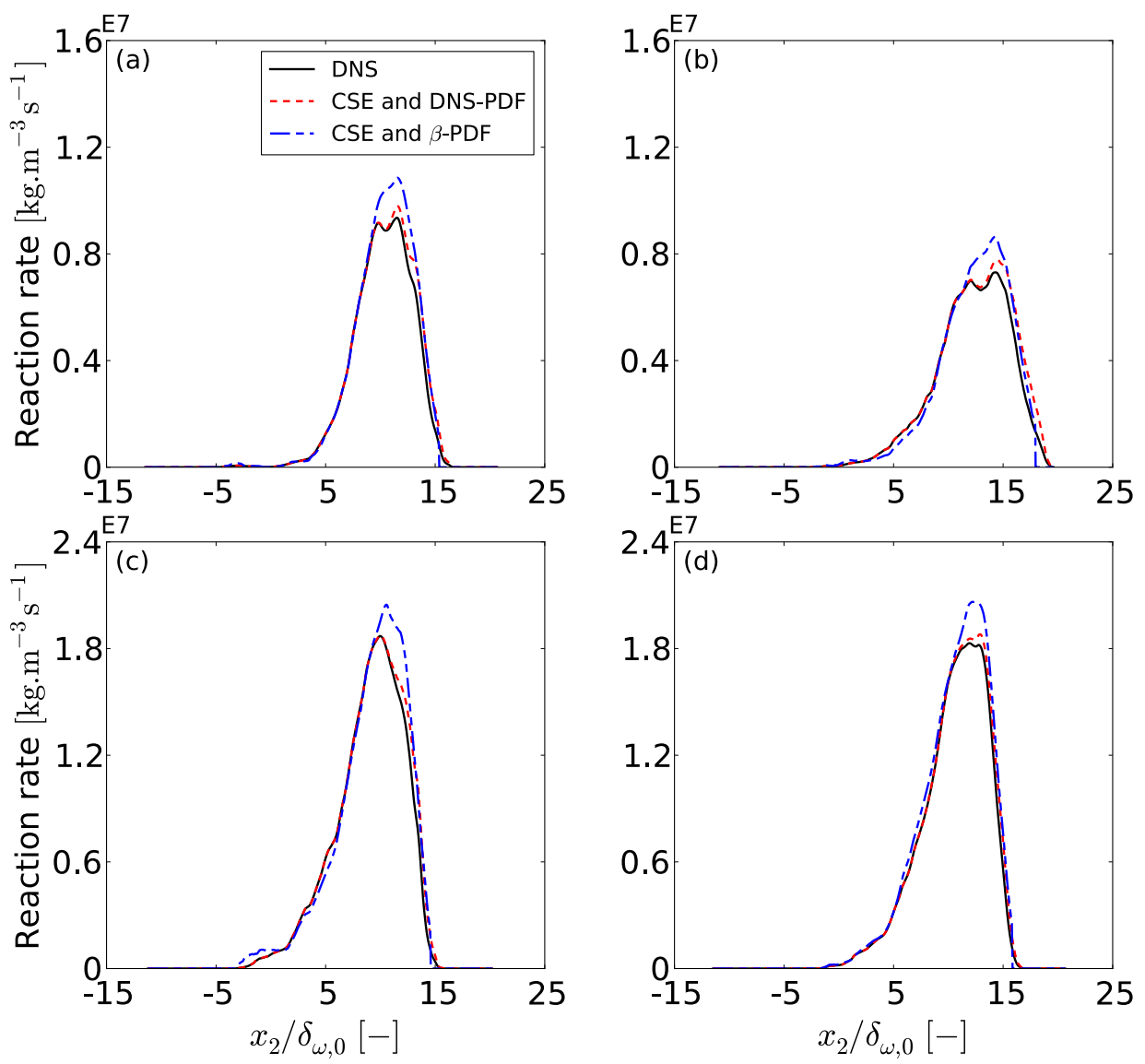

Fig. 6. Planar average $\dot{\omega}$ at the respective $t_{p p}^{*}$ for (a) R1000p60, (b) R2000p60, (c) R1000p80 and (d) R1000p60a. 

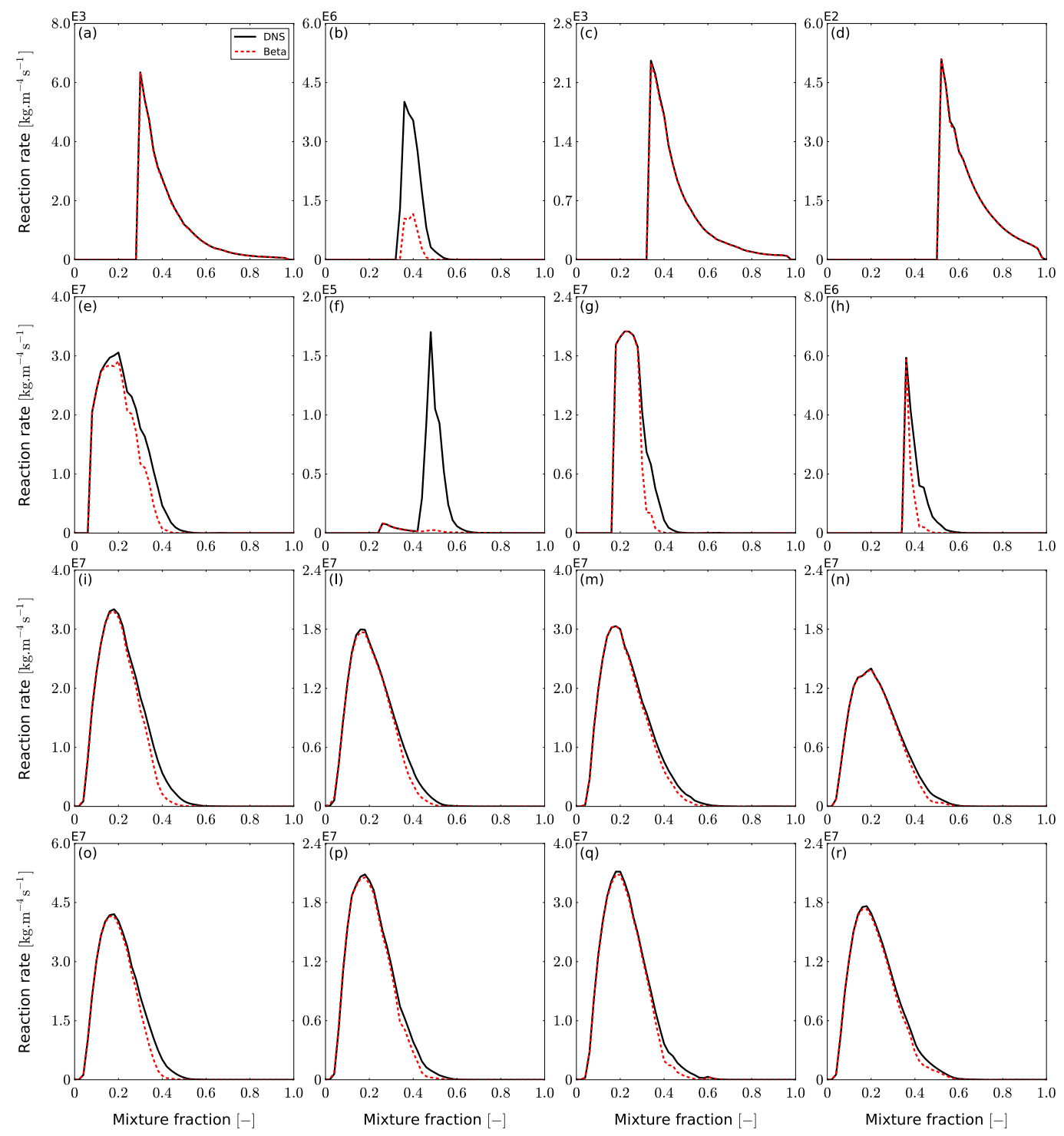

Fig. 7. Conditional $\dot{\omega}$ at different $x_{2} / \delta_{\omega, 0}$ locations, all at the respective $t_{p p}^{*}$. First column, R1000p80; second column, R1000p60; third column, R1000p60a; fourth column, R2000p60. First row, $x_{2} / \delta_{\omega, 0}=-4$; second row, $x_{2} / \delta_{\omega, 0}=0$; third row, $x_{2} / \delta_{\omega, 0}=8$; fourth row, $x_{2} / \delta_{\omega, 0}=12$. "DNS" refers to the computation performed according to the left hand side of Eq. (13). "Model" denotes the right hand side of Eq. (13). 